\title{
ASYMPTOTIC BEHAVIOR OF SOLUTIONS OF A FUNCTIONAL EQUATION ${ }^{1}$
}

\author{
J. R. BLUM, M. J. NORRIS AND G. M. WING
}

We consider the question as to whether or not the hypothesis that

$$
\lim _{t \rightarrow \infty}(f(t+s)-f(t))=\lambda s
$$

for all $s$ implies the conclusion that

$$
\lim _{t \rightarrow \infty} \frac{f(t)}{t}=\lambda
$$

in the presence of appropriate auxiliary hypotheses. We show that the result is not valid without some additional hypothesis; and that either measurability of $f$ or boundedness of $f$ on an appropriate set of intervals is adequate.

In the presence of auxiliary hypotheses, the requirement that (1) hold for all $s$ is apt to be unnecessarily strong. Theorem 1 covers a situation in which (1) is assumed for some nonzero $s$; while Theorems 2 and 3 cover situations, by reduction to Theorem 1, in which (1) is assumed for a more plentiful set but in which weaker side conditions are assumed.

Functions satisfying (1) arise in renewal theory [1]. In such cases, the functions usually satisfy stronger additional conditions than any we consider here, and (2) may often be obtained from such conditions instead of (1).

In what follows we assume that $f$ is a real-valued function defined on a set containing a right ray and $\lambda$ is a real number. It is clear that if (1) holds for $s$ in some set $S$, then (1) holds for $s$ in the additive subgroup of the real numbers generated by $S$; and we shall only consider such subgroups in our hypotheses.

THEOREM 1. Let $f$ be bounded on the finite subintervals of some right ray, and let (1) hold for $s$ in the subgroup $S$, where $S \neq\{0\}$. Then (2) holds.

Proof. Let $\epsilon$ be positive. Take $s$ as a positive element of $S$. Then there exists a positive $t_{1}$ such that

Presented to the Society, September 2, 1960 under the title Asymptotic behavior of solutions of a system of functional equations; received by the editors June 24, 1960 .

1 This work was performed under the auspices of the United States Atomic Energy Commission. 


$$
|f(t+s)-f(t)-\lambda s|<\epsilon
$$

We further restrict $t_{1}$ so that $f$ is bounded on finite subintervals of $\left[t_{1}, \infty\right)$. With brackets denoting integral part, we have

$$
t=\left[\left(t-t_{1}\right) / s\right] s+r,
$$

where $r \in\left[t_{1}, t_{1}+s\right)$. Let $M$ be a bound for $|f|$ on $\left[t_{1}, t_{1}+s\right)$.

Then, for $t \geqq t_{1}$, using (3) and the fact that $r \geqq t_{1}$,

$$
\begin{aligned}
& \left|\frac{f(t)}{t}-\lambda\right| \\
& =\left|\frac{\sum_{n=1}^{\left[\left(t-t_{1}\right) / s\right]}(f(n s+r)-f((n-1) s+r))+f(r)-\sum_{n=1}^{\left[\left(t-t_{1}\right) / s\right]} \lambda s-\lambda r \mid}{t}\right| \\
& =\left|\frac{\sum_{n=1}^{\left[\left(t-t_{1}\right) / s\right]}(f(n s+r)-f((n-1) s+r)-\lambda s)+f(r)-\lambda r \mid}{t}\right| \\
& \leqq \frac{\sum_{n=1}^{\left[\left(t-t_{1}\right) / s\right]}|f((n-1) s+r+s)-f((n-1) s+r)-\lambda s|+|f(r)|+|\lambda| r}{t} \\
& \leqq \frac{\left[\left(t-t_{1}\right) / s\right] \epsilon+M+|\lambda|\left(t_{1}+s\right)}{t} \leqq \frac{\epsilon}{s}+\frac{M+|\lambda|\left(t_{1}+s\right)}{t} .
\end{aligned}
$$

Hence

$$
\limsup _{t \rightarrow \infty}\left|\frac{f(t)}{t}-\lambda\right| \leqq \frac{\epsilon}{s} .
$$

Since $\epsilon$ was an arbitrary positive number, (2) holds.

THEOREM 2. Let $f$ be such that, given $t_{1}$, there exists a finite closed interval contained in $\left[t_{1}, \infty\right)$ on which $f$ is bounded. Let (1) hold for $s$ in the nondiscrete subgroup $S$. Then $f$ is bounded on the finite intervals of some right ray, and (2) holds.

Proof. Let $s_{1}$ be a positive element of $S$. Choose $t_{1}$ so that $\left|f\left(t+s_{1}\right)-f(t)-\lambda s_{1}\right|<1$ for $t \geqq t_{1}$. Choose a finite closed interval $I_{1}$ contained in $\left[t_{1}, \infty\right)$ on which $f$ is bounded. Then $f$ is also bounded on every translation of $I_{1}$ by a positive integral multiple of $s_{1}$.

Since $S$ is a nondiscrete group, we can choose $s_{2}$ in $S$ such that $s_{2}$ is positive and not greater than the length of $I_{1}$. Choose $t_{2}$ so that 
$\left|f\left(t+s_{2}\right)-f(t)-\lambda s_{2}\right|<1$ for $t \geqq t_{2}$. Let $I_{2}$ be a translation of $I_{1}$ by a positive integral multiple of $s_{1}$ such that $I_{2} \subset\left[t_{2}, \infty\right)$. Then $f$ is bounded on every translation of $I_{2}$ by a non-negative integral multiple of $s_{2}$. However, since $s_{2}$ is not greater than the length of $I_{2}$; such translations cover some right ray, and $f$ is bounded on the finite subintervals of this ray. Since $S$ is not discrete, $S \neq\{0\}$; and, by Theorem 1, (2) holds.

Before proving Theorem 3, which considers the case that $f$ is measurable, we prove several lemmas. In the proofs, in connection with words such as "measurability," "measure," and so forth, the related measure will be obvious.

Lemma. Let $\nu$ be Lebesgue outer measure for the real plane, let $A$ be $a$ set such that $\nu(A)>0$, and let $\alpha$ be less than 1. Then, for specified rectangular axes, there exists a square $W$ with sides parallel to the axes such that $\nu(A \cap W)>\alpha \nu(W)$.

Proof. With simple modifications the proof of $[3$, Theorem A, p. 68] carries through.

Leмma. If $\mu$ is Lebesgue outer measure for the real line, $A$ and $B$ are sets of real numbers not of $\mu$-measure zero, and $\alpha<1$; then there exist intervals $U$ and $V$ of equal length such that $\mu(A \cap U)>\alpha \mu(U)$ and $\mu(B \cap V)>\alpha \mu(V)$.

Proof. Let $\nu$ be Lebesgue outer measure for the real plane. Then $\nu(A \times B)=\mu(A) \mu(B)>0$. (Confer $[3$, p. 150, (9) ]). Hence, by the above lemma, there exists a square $W$, which can be considered to be $U \times V$ where $U$ and $V$ are intervals of equal length, such that

Then

$$
\nu((A \times B) \cap W)>\alpha \nu(W)=\alpha \mu(U) \mu(V) .
$$

$$
\begin{aligned}
\alpha \mu(U) \mu(V) & <\nu((A \times B) \cap W)=\nu((A \cap U) \times(B \cap V)) \\
& \leqq \nu((A \cap U) \times V)=\mu(A \cap U) \mu(V),
\end{aligned}
$$

and $\mu(A \cap U)>\alpha \mu(U)$. Similarly $\mu(B \cap V)>\mu(V)$.

Lemma. Let $\mu$ be Lebesgue outer measure for the real line. Let $A$ and $B$ be sets of real numbers, such that $A$ is $\mu$-measurable and neither set has $\mu$-measure zero. Then the set $\{a-b \mid a \in A, b \in B\}$ contains an interval.

Proof. Once the last lemma is established, the proof follows closely a proof for the case that $B=A$.

Choose $U$ and $V$ as in the last lemma for $\alpha=3 / 4$. Let $u_{0}$ and $v_{0}$ be the midpoints of $U$ and $V$, respectively, and let $l=\mu(U)=\mu(V)>0$. 
Consider the set $\left((A \cap U) \oplus v_{0}\right) \cup\left((B \cap V) \oplus\left(u_{0}+x\right)\right)$ where $|x|$ $<l / 2$. $(C \oplus D=\{r \mid c \in C, d \in D, r=c+d\}$, and similarly for other such notations). It is readily verified that this set is contained in the interval, $\left[u_{0}+v_{0}-l / 2+\min (0, x), u_{0}+v_{0}+l / 2+\max (0, x)\right]$, which has length $(l+|x|)$. Now $\mu\left((A \cap U) \oplus v_{0}\right)$ and $\mu\left((B \cap V) \oplus\left(u_{0}+x\right)\right)$ are greater than (3/4)l, and $(A \cap U) \oplus v_{0}$ is measurable; so that if the sets were disjoint, the measure of the union would be greater than $(3 / 2) l$, and therefore greater than $(l+|x|)$. To avoid this contradiction we must have that the two sets intersect, so there exist $a$ in $A$ and $b$ in $B$ such that

$$
\begin{aligned}
a+v_{0} & =b+u_{0}+x, \\
a-b & =u_{0}-v_{0}+x .
\end{aligned}
$$

Thus the set $\{a-b \mid a \in A, b \in B\}$ contains the interval $\left(u_{0}-v_{0}-l / 2, u_{0}-v_{0}+l / 2\right)$.

TheOREM 3. Let $\mu$ be Lebesgue outer measure for the real line. Let $f$ be such that, given $t_{1}$, there exists a $\mu$-measurable subset of positive $\mu$-measure contained in $\left[t_{1}, \infty\right)$ on which $f$ is $\mu$-measurable. Let (1) hold for $s$ in a subgroup $S$ with $\mu(S)>0$. Then, given $t_{1}$, there exists a finite closed interval contained in $\left[t_{1}, \infty\right)$ on which $f$ is bounded, and (2) holds.

Proof. Let $S^{\prime}=\{s \mid s \in S, 0<s<1\}$. Of course $\mu\left(S^{\prime}\right)>0$. For $n$ a positive integer, let $S_{n}=\left\{s\left|s \in S^{\prime}, t \geqq n \Rightarrow\right| f(t+s)-f(t)-\lambda s \mid<1\right\}$. Now $S^{\prime}=\cup_{n=1}^{\infty} S_{n}$, so $\mu\left(S^{\prime}\right) \leqq \sum_{n=1}^{\infty} \mu\left(S_{n}\right)$. Hence, we can take $n$ so that $\mu\left(S_{n}\right)>0$.

Now, given $t_{1} \geqq n$, let $A_{0}$ be a measurable set of positive measure contained in $\left[t_{1}, \infty\right)$ on which $f$ is measurable. For $m$ a positive integer the set $A_{m}, A_{m}=\left\{t\left|t \in A_{0},\right| f(t) \mid \leqq m\right\}$, is measurable and $f$ is bounded on it. Moreover, $A_{0}=\cup_{m=1}^{\infty} A_{m}$, so some $A_{m}$ has positive measure. Hence there is a measurable set $A$, of positive measure and contained in $\left[t_{1}, \infty\right)$, on which $f$ is bounded. Furthermore, $A \oplus S_{n} \subset\left[t_{1}, \infty\right)$, and $A \oplus S_{n}=A \ominus\left(\ominus S_{n}\right)$. Thus $A \oplus S_{n}$ contains an interval by the last lemma. Finally, for $t$ in $A$ and $s$ in $S_{n}$, we have $f(t), s$, and $|f(t+s)-f(t)-\lambda s|$ bounded. Hence $f(t+s)$ is bounded for $t$ in $A$ and $s$ in $S_{n}$, and $f$ is bounded on an interval contained in $\left[t_{1}, \infty\right)$.

Since $S$ has positive measure, it is nondenumerable and, therefore, nondiscrete. By Theorem 2, (2) holds.

We close with an example showing that some additional hypothesis is necessary to insure that the assumption of (1) for all $s$ implies (2).

THEOREM 4. There exists a function such that (1) holds for all s and (2) does not hold. 
Proof. Let $L$ be a real-valued additive function on the real numbers other than multiplication by a real number [2, Chapter III, $\S 9$, pp. 116-117].

Let $f$ be defined by

$$
f(t)=(t+|L(t)|)^{1 / 2}, \quad(t \geqq 1) .
$$

Now, for $t$ and $t+s$ greater than 1 ,

$$
\begin{aligned}
|f(t+s)-f(t)| & =\left|(t+s+|L(t+s)|)^{1 / 2}-(t+|L(t)|)^{1 / 2}\right| \\
& =\frac{|s+| L(t+s)|-| L(t)||}{(t+s+|L(t+s)|)^{1 / 2}+(t+|L(t)|)^{1 / 2}} \\
& =\frac{|s+| L(t)+L(s)|-| L(t)||}{(t+s+|L(t+s)|)^{1 / 2}+(t+|L(t)|)^{1 / 2}} \\
& \leqq \frac{|s|+|L(s)|}{(t)^{1 / 2}} .
\end{aligned}
$$

Hence $\lim _{t \rightarrow \infty}|f(t+s)-f(t)|=0 \cdot s$ for all $s$.

However, $L(t)$ is unbounded in every interval, [2, pp. 116-117]; so $f(t) / t$ must be unbounded in every interval. Thus $f(t) / t$ does not have limit zero as $t \rightarrow \infty$, and (2) does not hold.

\section{REFERENCES}

1. D. Blackwell, $A$ renewal theorem, Duke Math. J. vol. 15 (1948) pp. 145-150.

2. H. Hahn and A. Rosenthal, Set functions, Albuquerque, University of New Mexico Press, 1948.

3. P. R. Halmos, Measure theory, New York, Van Nostrand, 1950.

Sandia Corporation 